International Journal of Modern Physics E

Vol. 21, No. 10 (2012) 1292002 (2 pages)

(C) World Scientific Publishing Company

DOI: $10.1142 / \mathrm{S} 0218301312920024$

\title{
ERRATUM
}

\section{SOME DEFORMATION PROPERTIES OF THE EVEN-EVEN YTTERBIUM, HAFNIUM AND TUNGSTEN NUCLEI}

[INT. J. MOD. PHYS. E, Vol. 21, No. 9 (2012) 1250077]

S. B. DOMA* and H. S. EL-GENDY ${ }^{\dagger}$

Faculty of Science, Alexandria University, Moharram Bay, Alexandria, Egypt

*sbdoma@yahoo.com

†hselgendy1@yahoo.com

Received 23 September 2012

Published 2 October 2012

The following are corrections to errors in the published paper:

1. On page 3 , in line 26 , printed as

$\ldots$ the deformation parameters $\beta \gamma, \ldots$

should read

$\ldots$ the deformation parameters $\beta$ and $\gamma, \ldots$

2. On page 9 , in line 7 , printed as

$$
A=\frac{\hbar^{2}}{2 I}
$$

should read

$$
A=\frac{\hbar^{2}}{2 \Im}
$$

3. On page 9, in Eq. (42), printed as

$$
E_{\beta \text {-band }}=-\frac{\hbar^{2}}{J} \frac{1}{E_{\gamma}} I^{2}(I+1)^{2}+\frac{\hbar^{2}}{2 J} I(I+1)+E_{\beta} .
$$


should read

$$
E_{\beta \text {-band }}=-\frac{\hbar^{2}}{\Im} \frac{1}{E_{\gamma}} I^{2}(I+1)^{2}+\frac{\hbar^{2}}{2 \Im} I(I+1)+E_{\beta} .
$$

4. On page 9, in Eq. (43), printed as

$$
E_{\gamma \text {-band }}=\frac{\hbar^{2}}{2 \Im} \frac{1}{N} I^{2}(I+1)^{2}+\frac{1}{2} \frac{\hbar^{2}}{2 J} I(I+1)+E_{\gamma}
$$

should read

$$
E_{\gamma \text {-band }}=\frac{\hbar^{2}}{2 \Im} \frac{1}{N} I^{2}(I+1)^{2}+\frac{1}{2} \frac{\hbar^{2}}{2 \Im} I(I+1)+E_{\gamma}
$$

